Article

\title{
Cooperative Non-Orthogonal Multiple Access with Energy Harvesting
}

\author{
Weidong Guo * and Yunfeng Wang \\ College of Physics and Engineering, Qufu Normal University, Qufu 273165, Shandong, China; \\ wangyunfeng98@163.com \\ * Correspondence: gud2001@qfnu.edu.cn; Tel.: +86-537-445-6092
}

Received: 17 July 2017; Accepted: 12 September 2017; Published: 14 September 2017

\begin{abstract}
In this paper, we analyze the outage probability of an amplify-and-forward (AF) cooperative non-orthogonal multiple access (NOMA) model in multi-relay multiuser networks. In contrast to conventional cooperative networks, relays in the considered network have no embedded energy supply; they need to rely on the energy harvested from the signals broadcasted by the source for their cooperative NOMA transmission. Based on this structure, a new relay selection scheme is proposed, considering both channel state information (CSI) and battery status of relays. Assuming each relay has infinite or finite energy storage for accumulating energy, we use the infinite or finite Markov chain to capture the evolution of relay batteries and certain simplified assumptions to reduce computational complexity of the Markov chain analysis. The approximate closed-form expressions for the outage probability of the proposed scheme are derived therefrom. All theoretical results are validated by numerical simulations. The impacts of the system parameters, such as relay number, energy harvesting threshold and battery size, on the performance are extensively investigated.
\end{abstract}

Keywords: energy harvesting; non-orthogonal multiple access (NOMA); cooperative communication; Markov chain

\section{Introduction}

With the aim of increasing system capacity and achieving higher spectral efficiency, non-orthogonal multiple access (NOMA) has been recognized as a promising multiple access technique for fifth generation (5G) networks due to its superior spectral efficiency [1].The key idea of NOMA is to serve multiple users in the same frequency band, but with different power levels, which is a domain fundamentally different from conventional orthogonal access technologies [2]. In particular, power-domain NOMA allocates less transmitting power to users with better channel conditions and more transmit power to users with worse channel conditions in order to achieve a balanced tradeoff between system throughput and user fairness. Therefore, users can be separated by successive interference cancellation (SIC). Lan et al. [3] explored the impact of the error propagation of SIC and user velocity on the NOMA performance. Their results showed that, even in the worst error propagation scenario, NOMA outperforms conventional orthogonal multiple access and can yield performance gains for different user mobility. Chen et al. [4] studied NOMA for the downlink of a wireless system. Traditional minimum-mean-squared-error (MMSE) precoding matrices have been used. Considering that wireless relaying is an effective means to combat the effects of the fading and shadowing on transmitted signal, it is reasonable to combine NOMA with relaying networks. In [5], a new cooperative NOMA scheme was proposed and analyzed in terms of outage probability and diversity gain. The authors in [6] study the impact of relay selection on the performance of cooperative NOMA. In particular, a two-stage relay selection strategy is proposed; this strategy can achieve the minimal outage probability among all possible selection schemes, and realize the maximal diversity gain. 
In addition to improving spectral efficiency, which is the motivation of NOMA, another key objective of future $5 \mathrm{G}$ networks is to maximize energy efficiency. Energy harvesting (EH), a technique to collect energy from the surrounding environment, has recently received considerable attention as a sustainable solution to overcome the bottleneck of energy-constrained wireless sensor networks. Apart from the conventional renewable energy sources, such as solar and wind, radio frequency (RF) signals radiated by ambient transmitters can be treated as a viable new source for EH. Such an approach can reduce the cost of a sensor network, as peripheral equipment can be avoided [7]. However, a fundamental limitation of EH-based wireless communications lies in the restricted transmission range. Although longer ranges can be achieved through a stronger RF source, the available energy level to pick up at the distant receiver remains fairly small due to pathloss. In cellular and sensor networks, relays can be deployed to extend the coverage of base stations [8]. Recently, relays with $\mathrm{EH}$ capabilities have received much attention as they use the energy harvested from the source signal to perform information forwarding [9]. This can solve the problem of the energy supply of relays and expand the application of EH-based wireless communications. In [10], a wireless cooperative network is considered in which multiple source-destination pairs communicate with each other via an energy harvesting relay. In [11], both the source and the relay are EH devices and are charged by the destination serving as the power station. In [12], a novel best cooperative mechanism for wireless energy harvesting and spectrum sharing has been proposed, and this mechanism has been verified to be superior to the traditional schemes by simulation. The aforementioned literature concentrates on the achievable performance without considering energy storage at relays; i.e., the harvested energy within a transmission block is entirely consumed for forwarding information. Nevertheless, the energy harvested from RF radiation is often restricted, and thus it is desirable for relays to accumulate the harvested energy in energy storage such as super-capacitors or rechargeable batteries [13]. In [14], a threshold-based "save-then-transmit" scheme is employed at relays; the stored energy level in each relay battery actually forms a Markov chain over time. By investigating the properties of this Markov chain, the asymptotical average throughput is derived. In [15], to support an efficient utilization of harvested energy to improve throughput, a harvest-use-store relaying strategy with distributed beamforming has been researched.

The aforementioned three communication concepts, NOMA, EH and cooperative communication can be combined naturally, which is the focus of this paper. In particular, we consider a NOMA-based downlink amplify-and-forward (AF) relaying network. The main contributions of this paper are summarized as follows:

(1) We design a new NOMA-based relaying network and propose a new EH protocol for relays. The relays have no other energy supplies, but they are equipped with a chargeable battery, and thus can harvest and store the wireless energy broadcasted by the source. Then, we model the capacity of the relay battery in infinite and finite cases, respectively. The outage behavior of the network is investigated, and the closed-form expressions for the outage probability are derived.

(2) Simulation results are conducted to demonstrate our analytical results and the superiority of NOMA. In addition, the impacts of system parameters on the performance of the network are captured by simulation.

(3) Finally, we compared the proposed scheme with a conventional multiple access (MA) cooperative scheme. Simulation results show that, although conventional MA obtains better outage performance than our scheme, our scheme can offer better fairness, since more users are served simultaneously. In addition, our scheme can guarantee acceptable system performance (the best outage probability is $10^{-5}$ when transmission signal to noise ratio (SNR)is 20dB) even if the relays do not use their own batteries to power the relay transmission which demonstrates the superiority of our scheme compared to the common cooperative system or common NOMA system. 


\section{System Model}

As shown in Figure 1, a source $S$ and a number of potential users $D_{j}(j=1,2, \ldots, N)$ communicate over channels with flat fading. Multiple potential relays $f_{\left|h_{S R}\right|^{2}}(x)=\Omega \frac{1}{h_{1}} \sum_{k_{1}=0}^{\Omega-1}(-1)^{k_{1}}\left(\begin{array}{c}\Omega-1 \\ k_{1}\end{array}\right) \exp \left(\frac{-x\left(k_{1}+1\right)}{h_{1}}\right)$ are willing to amplify and forward the signal from $S$ to the users. $S$ has no direct link with users. The channels pertaining to the first hop and second hop undergo independent identical (i.i.d.) fading and the channel coefficients are denoted by $\widetilde{h}_{S R_{i}}$ and $\widetilde{h}_{R_{i} D_{j}}$, respectively. Assuming Rayleigh fading, $\widetilde{h}_{S R_{i}}$ is a circularly symmetric complex Gaussian random variable with zero mean and variance $\sigma_{S R}^{2}=\mathrm{E}\left(\left|\widetilde{h}_{S R_{i}}\right|^{2}\right)$. Likewise, $\sigma_{R D}^{2}=\mathrm{E}\left(\left|\widetilde{h}_{R_{i} D_{j}}\right|^{2}\right)$. The channel power gain $h_{S R_{i}}=\left|\widetilde{h}_{S R_{i}}\right|^{2}$ and $\theta_{n}^{*}=\max \left\{\theta_{1}, \theta_{2, \ldots} \theta_{n}\right\}$ thus follow the exponential distribution with mean $\sigma_{S R}^{2}$ and $\sigma_{R D}^{2}$. Without loss of generality, we assume that the users' channel gains have been ordered as $h_{R_{i} D_{1}} \leq h_{R_{i} D_{2}} \leq \cdots \leq h_{R_{i} D_{N}}$. We also assume that the additive white Gaussian noise (AWGN) of all links has a zero mean and equal variance $N_{0}$.

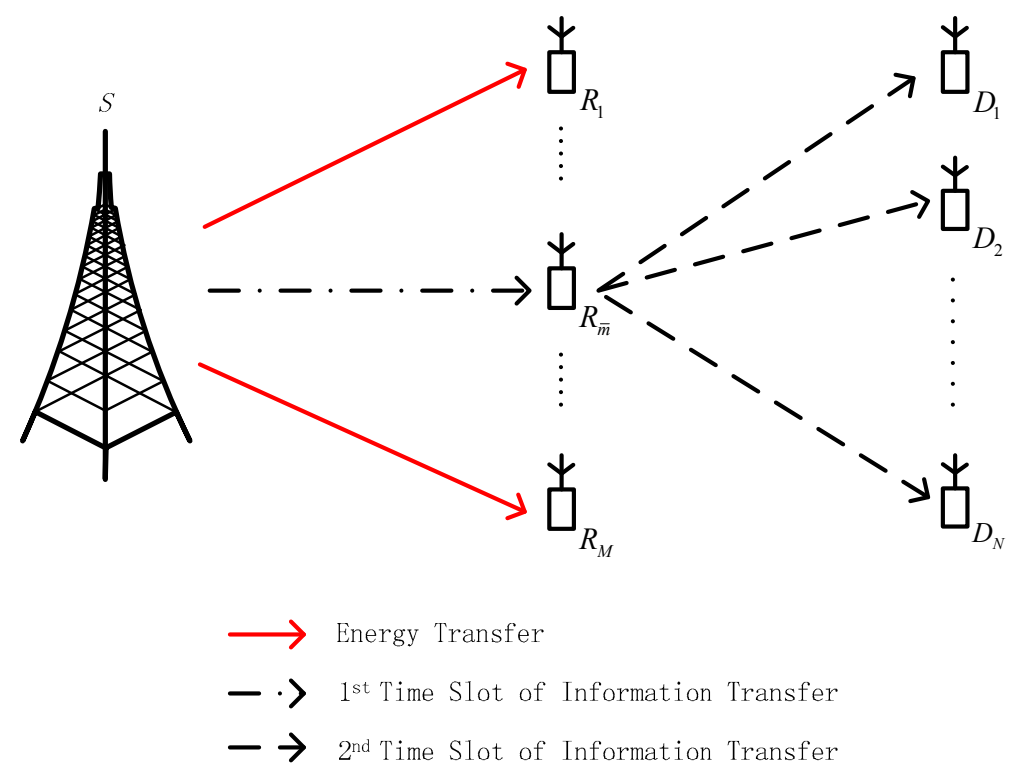

Figure 1. A reference model of system.

In this paper, we use the conventional AF protocol for the NOMA downlink cooperative network. From Figure 1, we can see that each transmission block takes two time slots; for convenience but without loss of generality, we consider a normalized unit block time (i.e., $T=1$ ) hereafter, so each time slot takes $T / 2$ and the energy harvesting time is also $T / 2$. Before the transmission, each relay checks their battery at the beginning of a transmission block and sees if it has enough energy to forward the source information. If the relay does not have enough energy, it performs EH in this time block and stores the harvested energy into the individual battery. We assume $z=y-\theta_{n}^{*}$ is the transmission power and is sufficiently large such that the energy harvested from the noise is negligible. Thus, the amount of energy harvested from the source can be expressed as [16]

$$
E_{R_{i}}=\eta P_{S} h_{S R_{i}} T / 2
$$

where $\eta$ is the energy harvesting efficiency. 
For those relays with sufficient energy, they report their CSI to $S$ for making the relay selection decision. Let $e_{i}$ denote the battery energy amount of relay $R_{i}$; we define those relays with enough energy as the eligible set

$$
\phi=\left\{R_{i} \mid e_{i} \geq W C, i=1,2, \ldots M\right\}
$$

where $C$ is the energy harvesting threshold to activate the $\mathrm{EH}$ circuit and $W$ represents the number of times transmit energy over $C$. Among this set of relays, $R_{\bar{m}}$ is selected, which can be expressed as

$$
\bar{m}=\arg \max _{i: R_{i} \in \phi}\left\{h_{S R_{i}}\right\}
$$

In Equation (3), the relay with the best channel power gain of the first hop in this set is selected for forwarding information.

The two-phase communication starts after relay selection. In the first slot, $S$ transmits the unit-power superposition symbol $x_{S}=\sum_{j=1}^{N} \sqrt{a_{j} P_{S}} x_{j}$ to the selected $R_{\bar{m}}$, where $x_{j}$ contains the information required by $D_{j}, a_{j}$ is the power allocation coefficient of $D_{j}$ which denotes the proportion of the transmit power allocated to $x_{j}$. Following the principle of NOMA, we have $a_{1}>a_{2} \cdots a_{N}$ and $\sum_{j=1}^{N} a_{j}=1$. The observation at $R_{\bar{m}}$ can be expressed as

$$
y_{R_{\bar{m}}}=\widetilde{h}_{S R_{\bar{m}}} \sum_{j=1}^{N} \sqrt{a_{j} P_{S}} x_{j}+n_{R_{\bar{m}}}
$$

where $n_{R_{\bar{m}}}$ is AWGN at $R_{\bar{m}}$. In this time slots, all other relays regard $x_{S}$ as energy signal and perform EH.

In the second slot, $R_{\bar{m}}$ amplifies $y_{R_{\bar{m}}}$ with an amplifying coefficient $\beta$ and then broadcasts it to all users. The signal received by the $n$th user can be expressed as

$$
y_{R_{\bar{m}} D_{n}}=\sqrt{P_{R}} \beta \widetilde{h}_{R_{\bar{m}} D_{n}} \widetilde{h}_{S R_{\bar{m}}} \sum_{j=1}^{N} \sqrt{a_{j} P_{S}} x_{j}+\sqrt{P_{R}} \beta \widetilde{h}_{R_{\bar{m}} D_{n}} n_{R_{\bar{m}}}+n_{D_{n}}
$$

where $P_{R}$ is the transmit power at relay and $P_{R}=W C /(T / 2), n_{D_{n}}$ is AWGN at $n$th user, and $\beta$ should be determined as follows:

$$
\beta=1 / \sqrt{\left(P_{S} h_{S R_{\bar{m}}}+N_{0}\right)}
$$

For each user, the desired signal is interfered by the other users' signals. SIC will be carried out at each user to mitigate the negative effect of the inter-user interference. The SIC decoding order is in increasing order of the effective users' channel gains $\left(h_{R_{\bar{m}} D_{1}} \leq h_{R_{\bar{m}} D_{2}} \leq \cdots \leq h_{R_{\bar{m}} D_{N}}\right)$. Therefore, at the $n$th user, the $l$ th user's signal, $l<n$, will be detected and then cancelled out from the received signal of the $n$th user in a successive manner. The $l$ th user's signal, $l>n$, will be treated as noise at the $n$th user. If we set $\gamma_{1}=P_{S} / N_{0}$ and $\gamma_{2}=P_{R} / N_{0}$, then, we can compute the signal to interference and noise ratio (SINR) for the $n$th user to decode the $l$ th user's signal, $l<n$, as follows:

$$
\gamma_{R_{\bar{m}} D_{l \rightarrow n}}=\frac{a_{l} \gamma_{1} \gamma_{2} h_{S R_{\bar{m}}} h_{R_{\bar{m}} D_{n}}}{\gamma_{1} \gamma_{2} h_{S R_{\bar{m}}} h_{R_{\bar{m}} D_{n}} \sum_{j=l+1}^{N} a_{j}+\gamma_{1} h_{S R_{\bar{m}}}+\gamma_{2} h_{R_{\bar{m}} D_{n}}+1}
$$

Accordingly, the instantaneous rate can be written as $R_{l \rightarrow n}=\frac{1}{2} \log _{2}\left(1+\gamma_{R_{\bar{m}} D_{l \rightarrow n}}\right)$. If the message $x_{l}$ can be decoded successfully, i.e., $R_{l \rightarrow n} \geq \widetilde{R}_{l}=\frac{1}{2} \log _{2}\left(1+\gamma_{\text {th }}^{l}\right)$, it will be removed from the $n$th user's observation, where $\widetilde{R}_{l}$ and $\gamma_{\text {th }}^{l}$ denote the target data rate and the target SINR for the $l$ th user, respectively. This SIC will be implemented until $\mathrm{n}$ users' messages are all decoded, where the SINR for the $n$th user to decode its own signal is given by

$$
\gamma_{R_{\bar{m}} D_{n}}=\frac{a_{n} \gamma_{1} \gamma_{2} h_{S R_{\bar{m}}} h_{R_{\bar{m}} D_{n}}}{\gamma_{1} \gamma_{2} h_{S R_{\bar{m}}} h_{R_{\bar{m}} D_{n}} \sum_{j=n+1}^{N} a_{j}+\gamma_{1} h_{S R_{\bar{m}}}+\gamma_{2} h_{R_{\bar{m}} D_{n}}+1}
$$


The $n$th user needs to decode all the other users' signals and the SNR for the $n$th user to decode its own signal can be expressed as

$$
\gamma_{R_{\bar{m}} D_{N}}=\frac{a_{N} \gamma_{1} \gamma_{2} h_{S R_{\bar{m}}} h_{R_{\bar{m}} D_{N}}}{\gamma_{1} h_{S R_{\bar{m}}}+\gamma_{2} h_{R_{\bar{m}} D_{N}}+1}
$$

It is obvious that $\gamma_{R_{\bar{m}} D_{n \rightarrow n}}=\gamma_{R_{\bar{m}} D_{n}}$ and $R_{n \rightarrow n}=R_{n}$. Hence, the achievable data rate for the $n$th user is given by $R_{n}=\frac{1}{2} \log _{2}\left(1+\gamma_{R_{\bar{m}} D_{n}}\right)$ conditioned on $R_{l \rightarrow n} \geq \widetilde{R}_{l}, l<n$.

\section{Performance Analysis}

In this section, the outage performance of the NOMA cooperative network will be characterized in terms of outage probability. According to the total probability law, the outage probability of the $n$th user can be written as

$$
P_{\text {out }}^{n}=\sum_{\Omega=1}^{M} \operatorname{Pr}(|\phi|=\Omega) \widetilde{P}_{\text {out }}^{n, \Omega}
$$

where $|\cdot|$ denotes the number of elements in a set and $\widetilde{P}_{\text {out }}^{n, \Omega}$ is the conditional outage probability when the number of $\phi$ is $\Omega$. We will first study the $\widetilde{P}_{\text {out }}^{n, \Omega}$ and then $\operatorname{Pr}(|\phi|=\Omega)$, finally, $P_{\text {out }}^{n}$ will be derived.

For ease of description, $\Lambda_{n, l}=\left\{R_{l \rightarrow n}<\widetilde{R}_{l}\right\}=\left\{\gamma_{R_{\bar{m}} D_{l \rightarrow n}}<\gamma_{\text {th }}^{l}\right\}, l \leq n$, is defined as the outage event where the $n$th user cannot decode the lth user's signal successfully, and $\Lambda_{n, l}^{c}$ is defined as the complementary set of $\Lambda_{n, l}$. As a result, the conditional outage probability for the $n$th user can be given by

$$
\widetilde{P}_{\text {out }}^{n, \Omega}=1-\operatorname{Pr}\left\{\Lambda_{n, 1}^{c} \cap \cdots \cap \Lambda_{n, n}^{c}\right\}
$$

To proceed forward, we first rewrite $\Lambda_{N, N}^{c}$ as

$$
\Lambda_{N, N}^{c}=\left\{\frac{a_{N} \gamma_{1} \gamma_{2} h_{S R_{\bar{m}}} h_{R_{\bar{m}} D_{N}}}{\gamma_{1} h_{S R_{\bar{m}}}+\gamma_{2} h_{R_{\bar{m}} D_{N}}+1}>\gamma_{\mathrm{th}}^{N}\right\}=\left\{h_{R_{\bar{m}} D_{n}}>\frac{\gamma_{\mathrm{th}}^{N}}{a_{N} \gamma_{2}} \triangleq \theta_{N}, h_{S R_{\bar{m}}}>\frac{\theta_{N}\left(1+\gamma_{2} h_{R_{\bar{m}} D_{N}}\right)}{\gamma_{1}\left(h_{R_{\bar{m}} D_{N}}-\theta_{N}\right)}\right\}
$$

Next, the other events $\Lambda_{n, l}^{c} l \neq N$, can be attained as

$$
\begin{aligned}
& \Lambda_{n, l}^{c}=\left\{\frac{a_{l} \gamma_{1} \gamma_{2} h_{S R_{\bar{m}}} h_{R_{\bar{m}} D_{n}}}{\left.\gamma_{1} \gamma_{2} h_{S R_{\bar{m}}} h_{R_{\bar{m}} D_{n} \sum_{j=l+1}^{N} a_{j}+\gamma_{1} h_{S R_{\bar{m}}}+\gamma_{2} h_{R_{\bar{m}} D_{n}}+1}>\gamma_{\text {th }}^{l}\right\}}\right. \\
& =\left\{h_{R_{\bar{m}} D_{n}}>\frac{\gamma_{\text {th }}^{l}}{\gamma_{2}\left(a_{l}-\gamma_{\text {th }}^{l} \sum_{j=l+1}^{N} a_{j}\right)} \triangleq \theta_{l}, h_{S R_{\bar{m}}}>\frac{\theta_{l}\left(1+\gamma_{2} h_{R_{\bar{m}} D_{n}}\right)}{\gamma_{1}\left(h_{R_{\bar{m}} D_{n}}-\theta_{l}\right)}\right\}
\end{aligned}
$$

From the final step of Equation (13) we can obtain a necessary condition is:

$$
a_{l} \geq \gamma_{\mathrm{th}}^{l} \sum_{j=l+1}^{N} a_{j}
$$

If the condition is not satisfied, the $n$th user can never decode the $l$ th user's signal successfully irrespective of the channel SINR.

By defining $\theta_{n}^{*}=\max \left\{\theta_{1}, \theta_{2}, \ldots \theta_{n}\right\}$, the outage probability for the $n$th user can be reformulated as

$$
\begin{aligned}
& \widetilde{P}_{\text {out }}^{n, \Omega}=1-\operatorname{Pr}\left\{h_{R_{\bar{m}} D_{n}}>\theta_{n}^{*}, h_{S R_{\bar{m}}}>\frac{\theta_{n}^{*}\left(1+\gamma_{2} h_{R_{\bar{m}} D_{n}}\right)}{\gamma_{1}\left(h_{\left.R_{\bar{m}} D_{n}-\theta_{n}^{*}\right)}\right)}\right. \\
& =\underbrace{\int_{0}^{\theta_{n}^{*}} f_{h_{R_{\bar{m}} D_{n}}}(y) d y}_{J_{1}}+\underbrace{\int_{\theta_{n}^{*}}^{\infty} f_{h_{R_{\bar{m}} D_{n}}}(y) F_{h_{S R_{\bar{m}}}}\left(\frac{\theta_{n}^{*}\left(1+\gamma_{2} y\right)}{\gamma_{1}\left(y-\theta_{n}^{*}\right)}\right) d y}_{J_{2}}
\end{aligned}
$$


Since $h_{S R_{i}}$ follows the exponential distribution with mean $\sigma_{S R}^{2}$, the probability density function (PDF) of $h_{S R_{\bar{m}}}$ can be written as

$$
f_{h_{S R_{\bar{m}}}}(x)=\Omega \frac{1}{\sigma_{S R}^{2}} \sum_{k_{1}=0}^{\Omega-1}(-1)^{k_{1}}\left(\begin{array}{c}
\Omega-1 \\
k_{1}
\end{array}\right) \exp \left(\frac{-x\left(k_{1}+1\right)}{\sigma_{S R}^{2}}\right)
$$

Similarly, the PDF of the ordered variable $h_{R_{\bar{m}} D_{n}}$ is given by

$$
f_{h_{R_{\bar{m}} D n}}(x)=N\left(\begin{array}{c}
N-1 \\
n-1
\end{array}\right) \frac{1}{\sigma_{R D}^{2}} \sum_{k_{2}=0}^{N-n}(-1)^{k}\left(\begin{array}{c}
N-n \\
k_{2}
\end{array}\right) \exp \left(\frac{-x\left(k_{2}+n\right)}{\sigma_{R D}^{2}}\right)
$$

In what follows, $J_{1}$ and $J_{2}$ will be addressed. Firstly, according to Equation (16), $J_{1}$ can be calculated as

$$
J_{1}=F_{h_{R_{\bar{m}} D}}\left(\theta_{n}^{*}\right)=N\left(\begin{array}{c}
N-1 \\
n-1
\end{array}\right) \sum_{k_{2}=0}^{N-n} \frac{(-1)^{k}}{k_{2}+n}\left(\begin{array}{c}
N-n \\
k_{2}
\end{array}\right)\left(1-\exp \left(\frac{-\theta_{n}^{*}\left(k_{2}+n\right)}{\sigma_{R D}^{2}}\right)\right)
$$

On the other hand, by substituting Equations (16) and (17) into $J_{2}$, it can be written as

$$
\begin{aligned}
& J_{2}=N \Omega\left(\begin{array}{c}
N-1 \\
n-1
\end{array}\right) \sum_{k_{1}=0}^{\Omega-1} \sum_{k_{2}=0}^{N-n} \frac{(-1)^{k_{1}+k_{2}}}{\left(k_{1}+1\right)\left(k_{2}+n\right)}\left(\begin{array}{c}
\Omega-1 \\
k_{1}
\end{array}\right)\left(\begin{array}{c}
N-n \\
k_{2}
\end{array}\right) \exp \left(\frac{-\theta_{n}^{*}\left(k_{2}+n\right)}{\sigma_{R D}^{2}}\right) \\
& \underbrace{-\frac{N \Omega\left(\begin{array}{c}
N-1 \\
n-1
\end{array}\right)}{\sigma_{R D}^{2}} \int_{\theta_{n}^{*}}^{\infty} \sum_{k_{1}=0}^{\Omega-1} \sum_{k_{2}=0}^{N-n} \frac{(-1)^{k_{1}+k_{2}}}{k_{1}+1}\left(\begin{array}{c}
\Omega-1 \\
k_{1}
\end{array}\right)\left(\begin{array}{c}
N-n \\
k_{2}
\end{array}\right) \exp \left(\frac{-y\left(k_{2}+n\right)}{\sigma_{R D}^{2}}+\frac{-\frac{\theta_{n}^{*}\left(1+\gamma_{2} y\right)}{\gamma_{1}\left(y-\theta_{n}\right)}\left(k_{1}+1\right)}{\sigma_{S R}^{2}}\right) d y}_{J_{3}}
\end{aligned}
$$

Defining $\frac{\left(k_{2}+n\right)}{\sigma_{R D}^{2}}=u$ and $\frac{\theta_{n}^{*}\left(k_{1}+1\right)}{\gamma_{1} \sigma_{S R}^{2}}=v, J_{3}$ can be attained as

$$
J_{3}=\frac{N \Omega\left(\begin{array}{c}
N-1 \\
n-1
\end{array}\right)}{\sigma_{R D}^{2}} \int_{\theta_{n}^{*}}^{\infty} \sum_{k_{1}=0}^{\Omega-1} \sum_{k_{2}=0}^{N-n} \frac{(-1)^{k_{1}+k_{2}}}{k_{1}+1}\left(\begin{array}{c}
\Omega-1 \\
k_{1}
\end{array}\right)\left(\begin{array}{c}
N-n \\
k_{2}
\end{array}\right) \exp \left(-u y+\frac{-v\left(1+\gamma_{2} y\right)}{\left(y-\theta_{n}^{*}\right)}\right) d y
$$

Defining $z=y-\theta_{n}^{*}$ and with the aid of Equation (3.478.4) in [17], $J_{3}$ can be calculated as follows

$$
J_{3}=\frac{2 N \Omega\left(\begin{array}{c}
N-1 \\
n-1
\end{array}\right)}{\sigma_{R D}^{2}} \exp \left(-u \theta_{n}^{*}-\gamma_{2} v\right) \sum_{k_{1}=0}^{\Omega-1} \sum_{k_{2}=0}^{N-n} \frac{(-1)^{k_{1}+k_{2}}}{k_{1}+1}\left(\begin{array}{c}
\Omega-1 \\
k_{1}
\end{array}\right)\left(\begin{array}{c}
N-n \\
k_{2}
\end{array}\right) \sqrt{\frac{v+\gamma_{2} v \theta_{n}^{*}}{u}} K_{1}\left(2 \sqrt{u\left(v+\gamma_{2} v \theta_{n}^{*}\right)}\right)
$$

Until now, we have been able to obtain a conditional exact closed-form expression for the conditional outage probability $\widetilde{P}_{\text {out }}^{n, \Omega}$ by substituting Equations (18) and (21) into (15). The next step is to get $\operatorname{Pr}(|\phi|=\Omega)$, which will be given in two cases based on energy storage capacity of relay.

\subsection{Infinite Storage of Energy}

In this case, we assume that each relay has infinite battery and define $C$ as the energy harvesting threshold to activate the EH circuit. Although harvested energy can be more than $C$ in one time slot, here, we consider each relay to only store $C$ and use the rest for its own purpose. The eligible set is composed by relays whose amount of harvested energy are no less than WC. We model the amount of the stored energy at each relay using an infinite state Markov chain as Figure 2. When the relay does not accumulate enough energy, i.e., the stored energy is smaller than $W C$, which corresponds to states $s=0,1,2 \ldots W-1$ in Figure 2, the relay cannot forward the information and there is no transition back to the previous states. When the relay has enough stored energy, i.e., the relay is in state $s \geq W$ in Figure 2 and is selected as the best relay, its stored energy state transits to state $s-W$ after the relaying. 


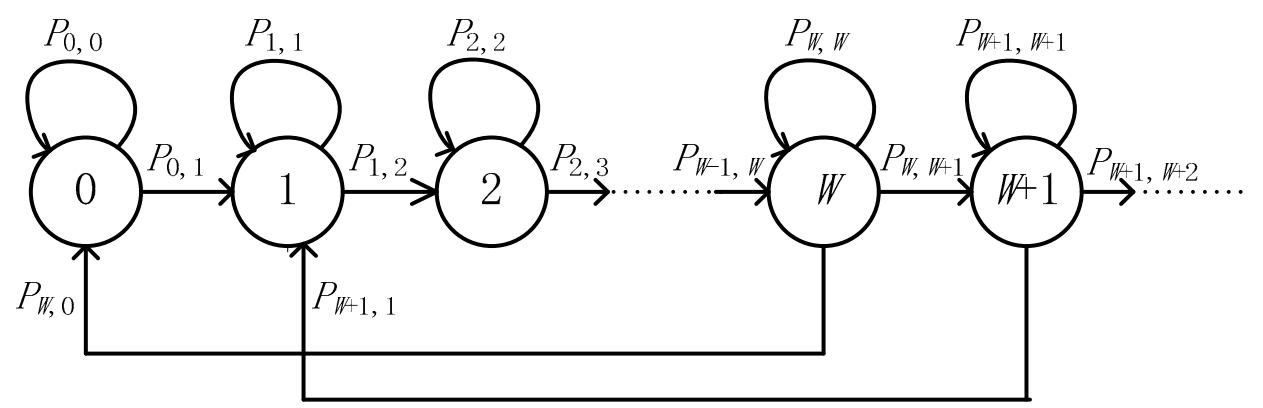

Figure 2. State transition diagram of the harvested energy amount. (The state $s$ denotes the relay has $s C$ amount of harvested energy at a relay.)

When $s<W$, the transition from state $s$ to state $s+1$ happens only when the relay harvest no less than $C$ amount of energy in one time slot. Hence, using Equation (1), we have

$$
P_{S, S+1}=\operatorname{Pr}\left(E_{R_{i}} \geq C\right)=\operatorname{Pr}\left(\eta P_{S} h_{S R_{i}} T / 2 \geq C\right)=\operatorname{Pr}\left(h_{S R_{i}} \geq \frac{2 C}{\eta P_{S} T}\right)=\exp \left(-\frac{2 C}{\eta P_{S} T \sigma_{S R}^{2}}\right)=\Lambda
$$

where $P_{a, b}$ is the transition probability from state $a$ to state $b$. Similarly, the transition from state $s$ to state $s$ happens when the relay performs $\mathrm{EH}$ but the harvested energy is not enough to increase its battery by one level, resulting in

$$
P_{s, s}=1-\operatorname{Pr}\left(E_{R_{i}} \geq C\right)=1-\Lambda
$$

When $s \geq W$, the transition from state $s$ to state $s+1$ happens when the relay harvest more than $C$ amount of energy in one time slot and this relay is not selected at this time slot. We note that the i.i.d. fading assumption implies each relay in $\phi$ has an equal chance to be selected as the best relay, and the average number of relays in $\phi$ is approximately equal to $M$ because of infinite storage capacity of relay. We denote $\operatorname{Pr}\left(R_{\bar{m}}=R_{i}\right)$ as the probability that $R_{i}$ is selected as the best relay, so $\operatorname{Pr}\left(R_{\bar{m}}=\right.$ $\left.R_{i}\right) \approx 1 / M$. This approximate expression may not always hold; we will discuss the effectiveness of the assumption through numerical results. Using this expression, the corresponding transition probability $P_{s, s+1}$ is given by

$$
P_{s, s+1}=\operatorname{Pr}\left(E_{R_{i}} \geq C\right)\left(1-\operatorname{Pr}\left(R_{\bar{m}}=R_{i}\right)\right) \approx \Lambda(1-1 / M)
$$

Similar to the above case, when $s \geq W$, the transition from state $s$ to state $s$ happens when the relay is not selected and the harvested energy is less than $C$ at this time slot. In this case, $P_{s, s}$ can be written as

$$
P_{S, S}=\left(1-\operatorname{Pr}\left(E_{R_{i}} \geq C\right)\right)\left(1-\operatorname{Pr}\left(R_{\bar{m}}=R_{i}\right)\right) \approx(1-\Lambda)(1-1 / M)
$$

If the relay which is in state $s \geq W$ is selected as the best relay for transmitting, its harvested energy then transits from state $s \geq W$ to state $s-W$. Hence, the transition probability $P_{s, s-W}$ is given by

$$
P_{S, S-W}=\operatorname{Pr}\left(R_{\bar{m}}=R_{i}\right) \approx 1 / M
$$


With the transition probabilities derived in Equations (22)-(26), we form an infinite dimensional transition matrix as $\mathrm{P}=\left[P_{a, b}\right](a, b \in \mathrm{N})$, which can be written as

$$
\mathrm{P}=\left[\begin{array}{ccccccccc}
p_{0,0} & p_{0,1} & 0 & \cdots & \cdots & \cdots & 0 & 0 & \cdots \\
0 & p_{1,1} & p_{1,2} & 0 & \cdots & \cdots & 0 & 0 & \cdots \\
\vdots & \vdots & \vdots & \vdots & \ddots & \cdots & 0 & 0 & \cdots \\
p_{W, 0} & 0 & 0 & 0 & \cdots & p_{W, W} & p_{W, W+1} & 0 & \cdots \\
0 & p_{W+1,0} & 0 & 0 & \cdots & 0 & p_{W+1, W+1} & p_{W+1, W+2} & \cdots \\
\vdots & \vdots & \vdots & \vdots & \vdots & \vdots & \vdots & \vdots & \ddots
\end{array}\right]
$$

Let $\mathrm{v}=\left(v_{1}, v_{2}, \ldots\right)$ denote the steady state probability vector; then, in the steady state, we have

$$
\mathrm{vP}=\mathrm{v}
$$

By expanding Equation (28) and making correspondence on both sides, we notice that

$$
\left\{\begin{array}{c}
p_{0,0} v_{0}+p_{W, 0} v_{N}=v_{0} \\
p_{0,1} v_{0}+p_{1,1} v_{1}+p_{W+1,1} v_{W+1}=v_{1} \\
\vdots \\
p_{k-1, k} v_{k-1}+p_{k, k} v_{k}+p_{W+k, k} v_{W+k}=v_{k} \\
\vdots
\end{array}\right.
$$

Based on the property of this transition matrix, we have

$$
\begin{gathered}
p_{k, k}+p_{k, k+1}=1, k<W \\
p_{k, k-W}+p_{k, k}+p_{k, k+1}=1, k \geq W
\end{gathered}
$$

Then, we have the following relation after some algebraic computation:

$$
p_{k, k+1} v_{k}=\left\{\begin{array}{l}
p_{k+W, k}\left(\sum_{j=0}^{k} v_{j+W}\right), 0 \leq k \leq W-1 \\
p_{k, k-W}\left(\sum_{j=1}^{W} v_{j+k}\right), k \geq W
\end{array}\right.
$$

Summing all the terms on both side of Equation (32), and using the fact that $\sum_{k=1}^{\infty} v_{k}=1$, we can obtain $v_{k}, \forall k \in \mathrm{N}$. Then, the probability that an relay can be in eligible set, is equal to the probability that the relay is in state $s \geq W$, which is given by

$$
\operatorname{Pr}(s \geq W)=\sum_{k=W}^{\infty} v_{k}=\frac{p_{0,1}}{W p_{k, k-W}-p_{k, k+1}+p_{0,1}}=\frac{\Lambda}{W-\Lambda(1-1 / M)+\Lambda}=\frac{M \Lambda}{W M+\Lambda}
$$

With the above result, we can get $\operatorname{Pr}(|\phi|=\Omega)$, which follows the binomial distribution with the probability mass function given as

$$
\operatorname{Pr}(|\phi|=\Omega)=\left(\begin{array}{c}
M \\
\Omega
\end{array}\right)(\operatorname{Pr}(s \geq W))^{\Omega}(1-\operatorname{Pr}(s \geq W))^{M-\Omega}
$$

With $\widetilde{P}_{\text {out }}^{n, \Omega}$ and Equation (34), we can get the close form expression of outage probability. 


\subsection{Finite Storage of Energy}

In this case, each relay accumulates the harvested energy using a finite energy storage with the size $B$. We also define $C$ as the energy harvesting threshold and $W C$ is the energy required for forwarding data, but relay can harvest $U C$ amount of energy in one time slot and $U$ is given by

$$
U C \leq E_{R_{i}}<(U+1) C, U \in \mathrm{N}
$$

This assumption is closer to the practical scenario, and the evolution of the battery status of all relays can be modeled as a finite state Markov chain; using the transition probability matrix of this chain, we can get the steady state probability vector and $\operatorname{Pr}(|\phi|=\Omega)$. Once $\operatorname{Pr}(|\phi|=\Omega)$ is obtained, the outage probability can also be obtained.

Due to the lack of general form of steady state probability, the above analysis is computationally intense when $M$ is large. To facilitate the computation, we propose an approximated approach based on two simplified assumptions. Firstly, we denote the relay energy amount at the selection epoch as a random variable $Z$. The exact distribution of $Z$ is high computational complexity. To ease the computation, we approximate $Z$ by a uniform random variable over $[0, B]$. The adopted approximation is inspired by considering the amount of harvested energy in a transmission block follows the geometric distribution with parameter $1 / 2$ [18]. In general, these conditions may not always hold. We will discuss the effectiveness of the assumption through numerical results. Secondly, we found that an arbitrary relay may be either short of enough power to participate in relay selection or otherwise, so the evolution of relay energy amount is captured by using two states, either active or inactive. With this simplified two-state Markov chain, a relay is in $s_{0}$ if the relay lacked sufficient energy to transmit, or in $s_{1}$ when the relay has enough energy for transmission. Next, we explain how to obtain the transition probability matrix of the two-state Markov chain.

The transition from state $s_{0}$ to state $s_{0}$ happens when a relay has no enough energy to transmit (i.e., $Z<W C$ ) in the current block and the accumulated energy after harvesting remains below WC. The corresponding transition probability is given by

$$
p_{0,0}=\operatorname{Pr}\left(Z+E_{R_{i}}<W C \mid 0 \leq Z<W C\right)=\operatorname{Pr}\left(\hat{Z}+E_{R_{i}}<W C\right)
$$

where $\hat{Z}$ is a truncated random variable defined as

$$
\hat{Z}= \begin{cases}Z, & Z<W C \\ 0, & Z \geq W C\end{cases}
$$

Since $Z$ is approximated as uniformly distributed, the PDF of $\hat{Z}$ can be obtained as

$$
f_{\hat{Z}}(z)=\frac{1}{B C} u(W C-z)+\left(1-\frac{W}{B}\right) \delta(z-W C)
$$

where $u(\cdot)$ and $\delta(\cdot)$ denote the unit step function and the Dirac delta function, respectively. Then Equation (36) can be solved as

$$
p_{0,0}=\int_{0}^{W C} \operatorname{Pr}\left(h_{S R}<\frac{W C-z}{\eta P_{S} T / 2}\right) f_{\hat{Z}}(z) d z=\frac{W}{B}-\frac{\bar{E}_{R_{i}}}{B C}\left(1-\exp \left(-\frac{W C}{\bar{E}_{R_{i}}}\right)\right)
$$

where $\bar{E}_{R_{i}}=\mathrm{E}\left(E_{R_{i}}\right)=P_{S} \eta \sigma_{S R}^{2} T / 2$.

The transition from $s_{0}$ to $s_{1}$ happens when the relay enters the EH mode in the current bock and the accumulated energy exceeds $W C$. Hence, we have

$$
p_{0,1}=\operatorname{Pr}\left(Z+E_{R_{i}} \geq W C \mid 0 \leq Z<W C\right)=\operatorname{Pr}\left(\hat{Z}+E_{R_{i}} \geq W C\right)
$$


Similar to the derivation of Equation (36), we obtain

$$
p_{0,1}=\frac{\bar{E}_{R_{i}}}{B C}\left(1-\exp \left(-\frac{W C}{\bar{E}_{R_{i}}}\right)\right)+\frac{B-W}{B}
$$

If the relay which is in state $s_{1}$ is selected as the best relay for transmitting, its harvested energy then transits from state $s_{1}$ to state $s_{0}$. Hence, the transition probability $p_{1,0}$ is given by

$$
p_{1,0}=\operatorname{Pr}(Z-W C<W C \mid W C \leq Z \leq B C) \operatorname{Pr}\left(R_{\bar{m}}=R_{i}\right)=\operatorname{Pr}(\widetilde{Z}<2 W C) \operatorname{Pr}\left(R_{\bar{m}}=R_{i}\right)
$$

where $\widetilde{Z}$ is a truncated random variable defined as

$$
\widetilde{Z}=\left\{\begin{array}{l}
Z, W C \leq Z \leq B C \\
0, Z<W C
\end{array}\right.
$$

Since $Z$ is uniformly distributed, the PDF of $\widetilde{Z}$ can be obtained as

$$
f_{\widetilde{Z}}(z)=\frac{1}{B C}(u(z-B C)-u(z-W C))+\frac{W}{B} \delta(z-W C)
$$

Using this PDF, the first term in the right of Equation (42) can be obtained as

$$
\operatorname{Pr}(\widetilde{Z}<2 W C)=\left\{\begin{array}{cc}
\frac{2 W}{B} & 2 W<B \\
1 & 2 W \geq B
\end{array}\right.
$$

As to the second term, we note that the i.i.d. fading assumption implies each relay in $\phi$ has an equal chance to be selected as the best relay. To simplify the analysis, we approximate the cardinality of $\phi$ by its mean such that

$$
\operatorname{Pr}\left(R_{\bar{m}}=R_{i}\right) \approx \frac{1}{\bar{M}_{e}}=\frac{1}{M v_{1}}
$$

where $\bar{M}_{e}$ is the average number of relays in $\phi$ and $v_{1}$ is the steady-state probability of state $s_{1}$. Combining Equations (45) and (46), we can obtain the closed-form for $p_{1,0}$.

The transition probability from $s_{1}$ to $s_{1}$ can be solved in the similar manner as the previous case, so we omit the derivation here.

When the two-state Markov chain formulated, the steady state probability vector can be easily obtained as

$$
v=\left(v_{0}, v_{1}\right)=\left(\frac{p_{1,0}}{p_{0,1}+p_{1,0}}, \frac{p_{0,1}}{p_{0,1}+p_{1,0}}\right)
$$

We note that both $v_{0}$ and $v_{1}$ involve $p_{1,0}$, which is a function of $v_{1}$. By substituting $p_{1,0}$ and $p_{1,0}$ into Equation (47), $v_{1}$ can be solved explicitly. Take the condition $2 W<B$ in Equation (45), for example; $v_{1}$ can be obtained in closed form as

$$
v_{1}=1-\frac{2 W}{p_{0,1} B M}=1-\frac{2 W}{B M}\left(\frac{\bar{E}_{R_{i}}}{B C}\left(1-\exp \left(-\frac{W C}{\bar{E}_{R_{i}}}\right)\right)+\frac{B-W}{B}\right)^{-1}
$$

With $v_{1}$ at hand, we can get $\operatorname{Pr}(|\phi|=\Omega)$, which follows the binomial distribution with the probability mass function given as

$$
\operatorname{Pr}(|\phi|=\Omega)=\left(\begin{array}{c}
M \\
\Omega
\end{array}\right)\left(v_{1}\right)^{\Omega}\left(1-v_{1}\right)^{M-\Omega}
$$

Submitting $\operatorname{Pr}(|\phi|=\Omega)$ into Equation (10), we can get outage probability of the system. 


\section{Simulation Results}

In this section, computer simulations are performed to validate the mentioned theoretical analysis. In all simulations, we set the noise power to $N_{0}=1$, the energy harvesting efficiency to $\eta=0.5$, and the fixed transmission rate of the source is 1 bit per channel use (bpcu). The battery size $B$ is set to be a multiple of the energy harvesting threshold $C$, i.e., $B=\alpha C$, where $\alpha \in N$ and $\alpha>W$. We also set $C$ is the multiple of the source transmission energy, i.e., $C=\delta P T / 2$, where $\delta>0$ is the scaling factor and the length of a transmission block $T=1$. To facilitate the analysis, $\sigma_{S R}^{2}$ and $\sigma_{R D}^{2}$ are set to be 1 . Without loss of generality, we set $N=3$, the power allocation coefficient are $a_{1}=1 / 2, a_{2}=1 / 3$ and $a_{3}=1 / 6$, the outage threshold are $\gamma_{\text {th }}^{1}=0.9 \mathrm{~dB}, \gamma_{\text {th }}^{2}=1.5 \mathrm{~dB}$ and $\gamma_{\text {th }}^{3}=2 \mathrm{~dB}$.

Figures 3 and 4 illustrate the performance of the proposed protocol with infinite battery size and finite battery size versus the transmitting SNR of source for given values of $C$ and $W$, respectively. We set $W=4$ and $\delta=0.5$. In Figures 3 and 4 , the approximated analysis of infinite and finite Markov chain is accurate when the number of relays is sufficient, but its results become deviated from thesimulated ones for $M=3$. The outage probability reveals an error floor when $M=3$. This is because, when $M=3$, relays cannot maintain a enough energy state in the infinite storage case, and the energy distribution of the relay battery is no longer as uniform as assumed in the finite storage case, respectively. However, when $M$ increases, the approximate cardinality of $\phi$ becomes more accurate. We can also see that the slope of the outage probability curve (namely, diversity order) increases with $M$. Since the theoretical analyses agree well with the simulations in medium and high SNR ranges, we will only plot the analytical results in the remaining figures.

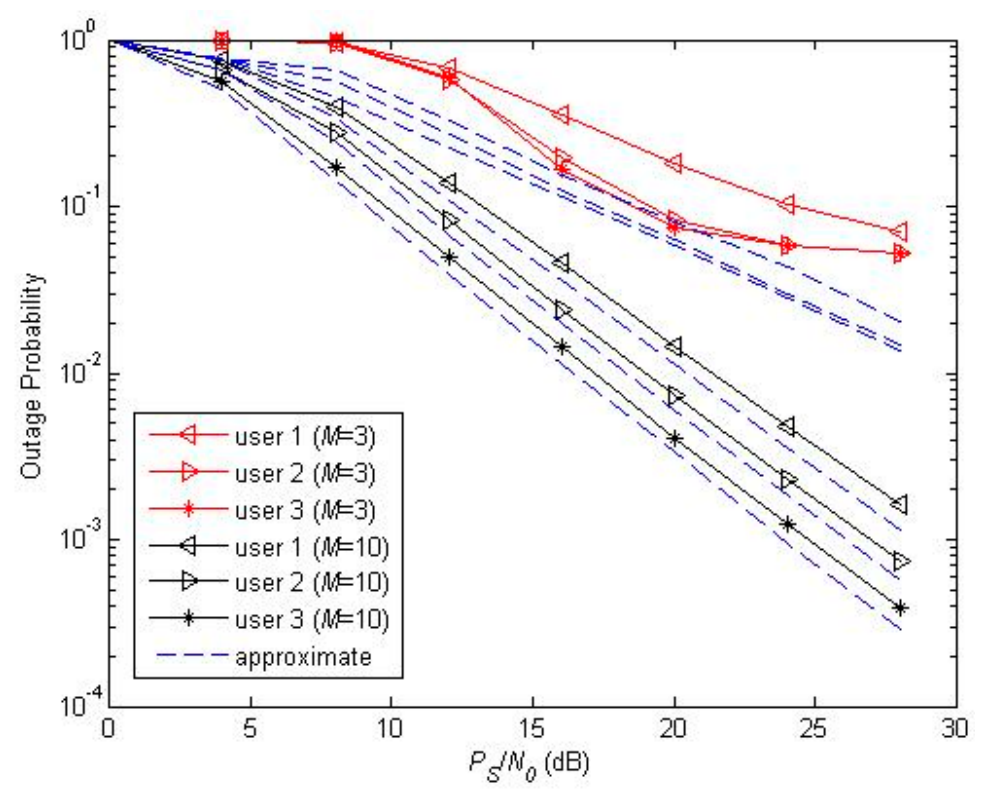

Figure 3. Outage probability with different $M$ (infinite storage of energy).

Next, we will investigate the impacts of the system parameters on the performance in medium SNR conditions $\left(P_{S} / N_{0}=20 \mathrm{~dB}\right)$. In Figures 5 and 6 , we illustrate the impacts of $C$ on the performance of the proposed two battery scheme with different $W . M=6$ is set in both two figures. For all the curves in Figures 5 and 6, the trends are the same for all schemes; namely, the probability first decreases then increases as $\delta$ varies from 0 to 1 . This means that, when the other parameters are determined, there must be an optimal value of $\delta$. However, the values of the inflection points are not always the same for different users and are inversely proportional to $W$. When $W=1$, the eligible set has more relays than $W=3$; the probability of choosing the best relay with more energy is larger than $W=3$, and so the optimal value of $\delta$ can be greater than $W=3$. The optimal value of $\delta$ can easily be 
obtained by a one-dimensional exhaustive search; with this optimal value of $\delta$, the system can resist fading more effectively.

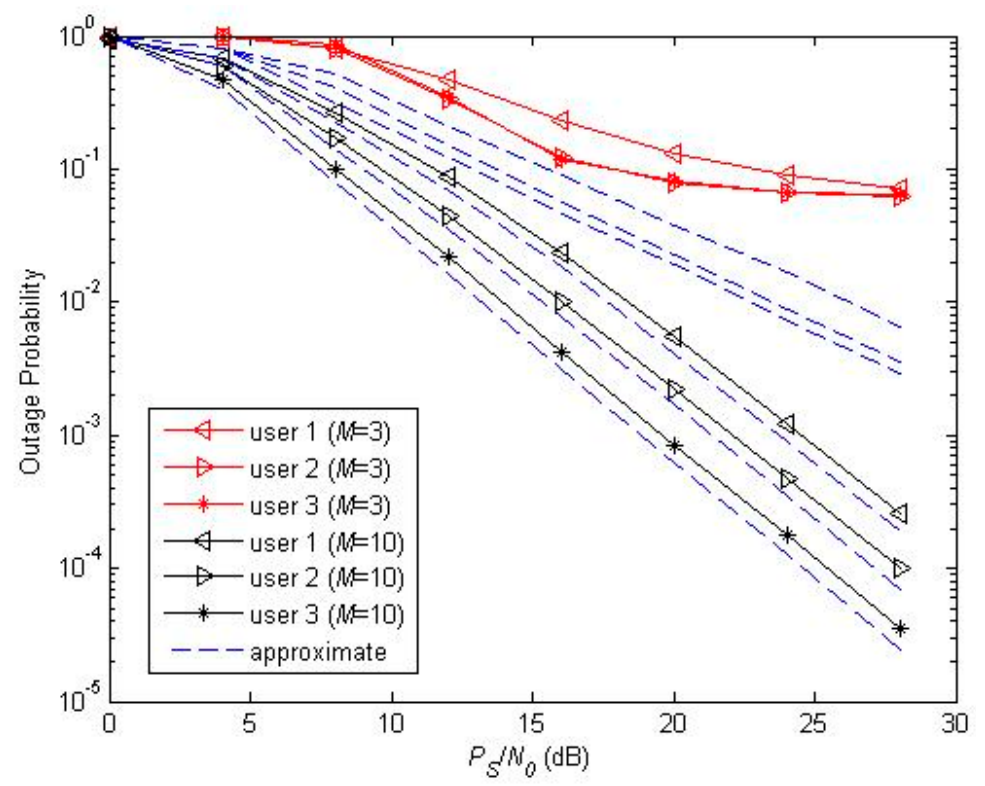

Figure 4. Outage probability with different $M$ (finite storage of energy, $\alpha=15$ ).

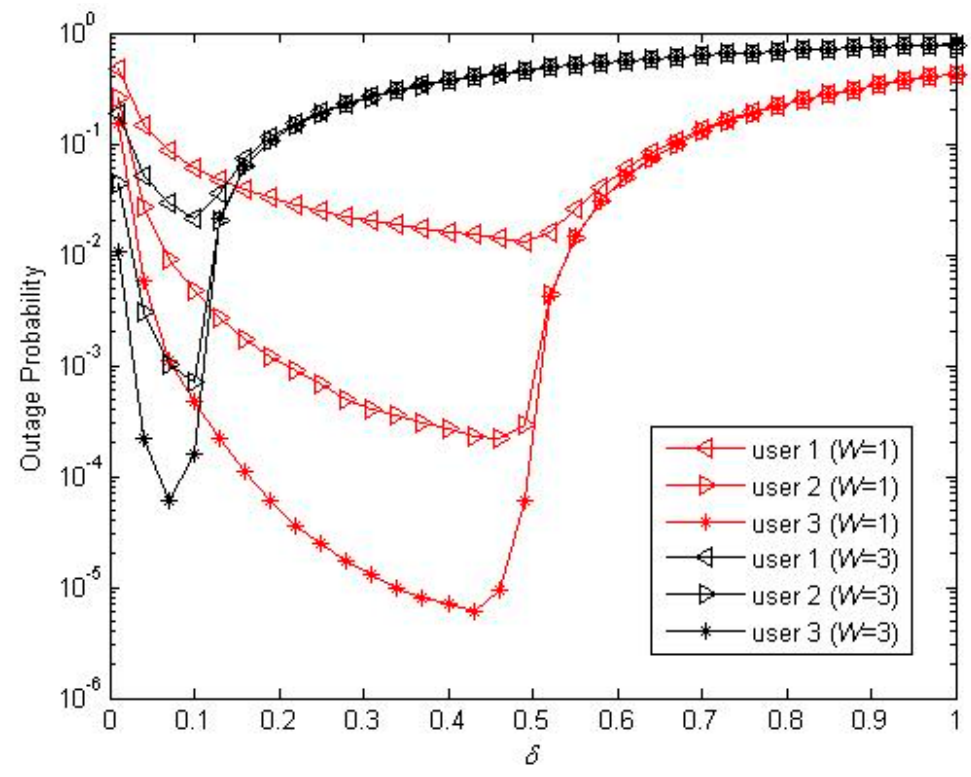

Figure 5. Outage probability with different $W$ (infinite storage of energy).

In Figure 7, we investigate the impact of battery size on the proposed finite battery scheme by varying the battery scaling factor with fixed number of relay $(M=6)$. Since three users have the same trend, we only analyze user 3 in this figure. From Figure 7, we can observe that the performance increases as $\alpha$ increases. However, the gain provided by a larger battery size does not increase when $\alpha$ exceeds a certain value and this value is decided by $W$. From this figure, we can also see that the order of performance for different $W$ changes when the value of $B$ changes. 


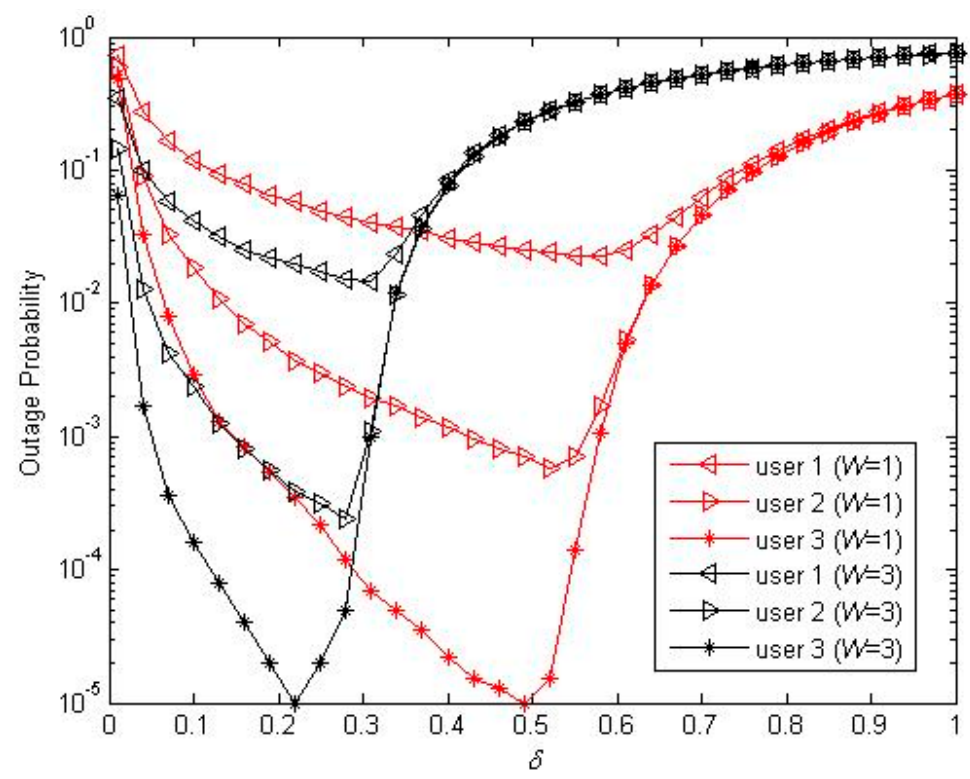

Figure 6. Outage probability with different $W$ (finite storage of energy, $\alpha=15$ ).

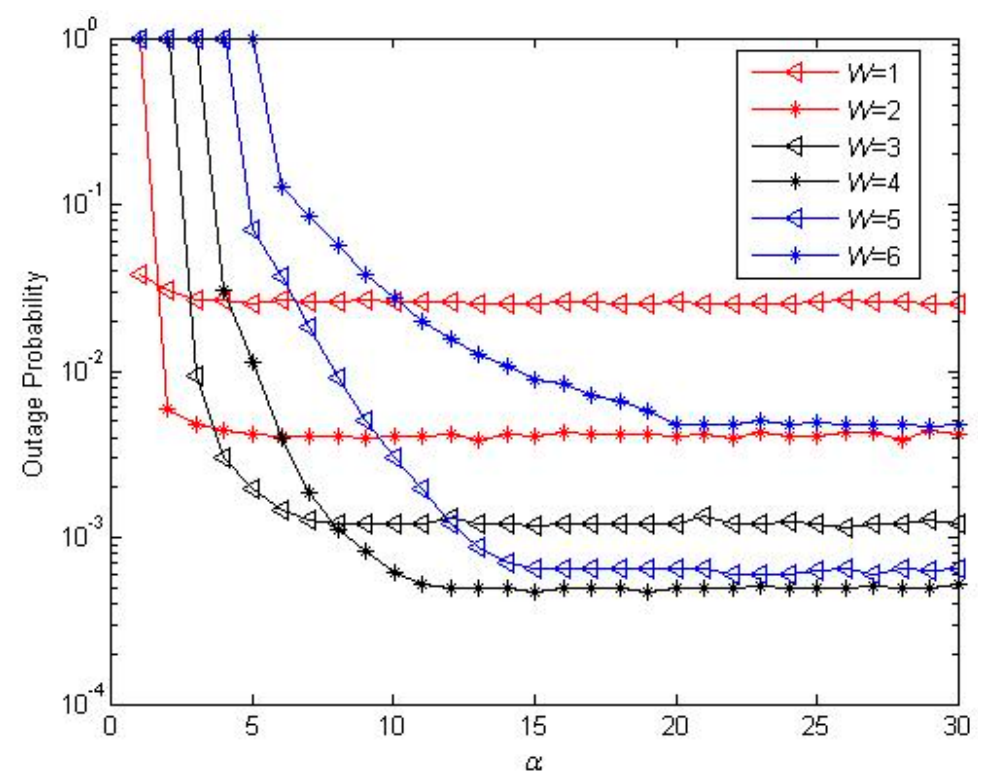

Figure 7. Outage probability with different $B(\delta=0.5)$.

Figures 8 and 9 illustrate the performance of the proposed protocol for different relay number. We also only analyze user 3 in both two figures. $W=4$ and $\delta=0.5$ are set. In order to illustrate the scalability of our algorithm, we set $M=3, M=5, M=10, M=20$ and $M=30$, respectively. From those two figures, we can see that, when $M$ is small, there is a huge drop of outage probability with a slight increase of $M$; this is because relays in the eligible set are insufficient when $M$ is small, and the slight increase of $M$ will increase the number of relays in the eligible set remarkably and a better relay can be selected. However, when $M$ is huge $(M=10$ or $M=20)$, relays in the eligible set are sufficient, and the increase of $M$ enhances the performance slightly, thus our algorithm can be used when $M$ is not more than 10. In addition, we compared the proposed scheme with conventional MA cooperative scheme. Here, we assume that an opportunistic MA approach is adopted for conventional MA, where the user with the best channel condition is scheduled. The target SINR $\gamma_{\text {th }}$ for conventional scheme satisfies $\frac{1}{2} \sum_{i=1}^{3} \log _{2}\left(1+\gamma_{\text {th }}^{i}\right)=\frac{1}{2} \log _{2}\left(1+\gamma_{\text {th }}\right)$ Simulation results show that conventional MA 
can achieve the same diversity gain with user 3. Conventional MA obtains better outage performance than NOMA, but NOMA can offer better fairness since more users are served simultaneously.

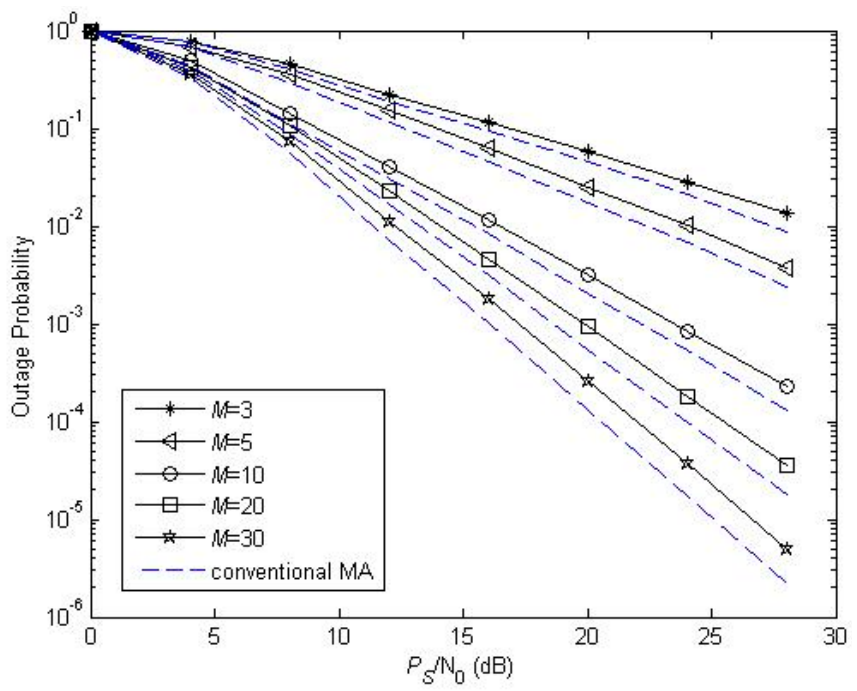

Figure 8. The impact of relay number on the outage probability (infinite storage of energy).

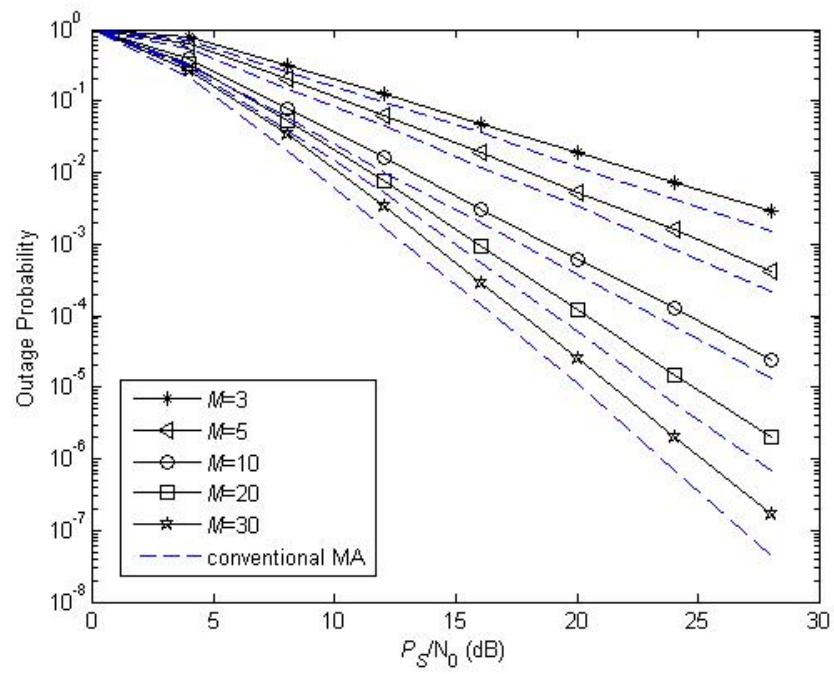

Figure 9. The impact of relay number on the outage probability (finite storage of energy, $\alpha=15$ ).

\section{Conclusions}

In this paper, we analyze the performance of the cooperative NOMA network. A new relay selection scheme is proposed considering both CSI and battery status of relays. We model the amount of harvested energy at each relay using an infinite or finite Markov chain and then derive the approximate closed-form expression of the outage probability in two cases, respectively. Simulations are carried out to verify the correctness of the theoretical analysis. We conclude the superiority of NOMA and find that, by carefully choosing the parameters of the network, (e.g., energy harvesting threshold or forwarding threshold), acceptable system performance can be guaranteed even if the relays do not use their own batteries to power the relay transmission. For future work, we will investigate the performance of cooperative NOMA in large-scale relaying networks using stochastic geometry.

Acknowledgments: This work was supported by the Scientific Research Initial Fund of Qufu Normal University (No. BSQD2012055), Youth Fund of Qufu Normal University (No. xkj201306) and Project of Shandong Province Higher Educational Science and Technology Program (No. J15LN57). 
Author Contributions: Weidong Guo conceived of the idea. He developed the mathematical models and performed the Monte Carlo simulations. Yunfeng Wang checked the mathematical model and the simulated results.

Conflicts of Interest: The authors declare no conflict of interest.

\section{References}

1. Saito, Y.; Benjebbour, A.; Kishiyama, Y.; Nakamura, T. System level performance evaluation of downlink non-orthogonal multiple access (NOMA). In Proceedings of the IEEE Annual International Symposium on Personal, In door and Mobile Radio Communications (PIMRC), London, UK, 8-11 September 2013; pp. 611-615.

2. Ding, Z.; Yang, Z.; Fan, P.; Poor, H.V. On the performance of non-orthogonal multiple access in 5G systems with randomly deployed users. IEEE Signal Process. Lett. 2014, 21, 1501-1505. [CrossRef]

3. Lan, Y.; Benjebboiu, A.; Chen, X.; Li, A.; Jiang, H. Considerations on downlink non-orthogonal multiple access (NOMA) combined with closed-loop SU-MIMO. In Proceedings of the IEEE International Conference on Signal Process and Communication System (ICSPCS), Gold Coast, Australia, 15-17 December 2014; pp. 1-5.

4. Chen, X.; Benjebbour, A.; Lan, Y.; Li, A.; Jiang, H. Impact of rank optimization on downlink non-orthogonal multiple access (NOMA)with SU-MIMO. In Proceedings of the IEEE International Conference on Communication System (ICCS), Macau, China, 19-21 November 2014; pp. 233-237.

5. Ding, Z.; Peng, M.; Poor, H.V. Cooperative non-orthogonal multiple access in 5G systems. IEEE Commun. Lett. 2015, 19, 1462-1465. [CrossRef]

6. Ding, Z.; Dai, H.; Poor, H.V. Relay selection for cooperative NOMA. IEEE Wirel. Commun. Lett. 2016, 5, 416-419. [CrossRef]

7. Liu, L.; Zhang, R.; Chua, K.C. Wireless information transfer with opportunistic energy harvesting. IEEE Trans. Wirel. Commun. 2013, 12, 288-300. [CrossRef]

8. Scaglione, A.; Goeckel, D.L.; Laneman, J.N. Cooperative communications in mobile ad hoc networks. IEEE Signal Process. Mag. 2006, 23, 18-29. [CrossRef]

9. Nasir, A.A.; Zhou, X.; Durrani, S.; Kennedy, R.A. Relaying protocols for wireless energy harvesting and information processing. IEEE Trans. Wirel. Commun. 2013, 12, 3622-3636. [CrossRef]

10. Ding, Z.; Perlaza, S.M.; Esnaola, I.; Poor, H.V. Power allocation strategies in energy harvesting wireless cooperative networks. IEEE Trans. Wirel. Commun. 2014, 13, 846-860. [CrossRef]

11. Chen, H.; Li, Y.; Rebelatto, J.L.; Uchôa-Filho, B.F.; Vucetic, B. Harvest-then-cooperate: Wireless-powered cooperative communications. IEEE Trans. Signal Process. 2015, 63, 1700-1711. [CrossRef]

12. Gao, H.; Ejaz, W.; Jo, M. Cooperative Wireless Energy Harvesting and Spectrum Sharing in 5G Networks. IEEE Access 2016, 4, 3647-3658. [CrossRef]

13. Sudevalayam, S.; Kulkarni, P. Energy harvesting sensor nodes: Survey and implications. IEEE Commun. Surv. Tutor. 2011, 13, 443-461. [CrossRef]

14. Huang, C.; Zhang, J.; Zhang, P.; Cui, S. Threshold-based transmissions for large relay networks powered by renewable energy. In Proceedings of the IEEE Global Communications Conferences (ICC), Budapest, Hungary, 9-13December 2013; pp. 1921-1926.

15. Zhou, Z.; Peng, M.; Zhao, Z.; Wang, W.; Blum, R.S. Wireless-powered cooperative communications: Power-splitting relaying with energy accumulation. IEEE J. Sel. Areas Commun. 2016, 34, 969-982. [CrossRef]

16. Ju, H.; Zhang, R. Throughput maximization in wireless powered communication networks. IEEE Trans. Wirel. Commun. 2014, 13, 418-428. [CrossRef]

17. Gradshteyn, I.; Ryzhik, I. Table of Integrals, Series and Products, 7th ed.; Academic Press: San Diego, CA, USA, 1994.

18. Rego, V. Characterization of equilibrium queue length distributions in M/GI/I queues. Comput. Oper. Res. 1988, 15, 7-17. [CrossRef]

(C) 2017 by the authors. Licensee MDPI, Basel, Switzerland. This article is an open access article distributed under the terms and conditions of the Creative Commons Attribution (CC BY) license (http:/ / creativecommons.org/licenses/by/4.0/). 